\title{
Enhancement the performance of an electro- optic switch by analysis the effect of tensile stress, axial and radial strain
}

\author{
Sadeq Adnan Hbeeb \\ Communication Engineering Department, Engineering College, University of Diayla \\ Sadeq.Hbeeb@yahoo.com
}

\begin{abstract}
This research presents a technique of an electro optic effect for suggestion model and it optimizes implementation of an electro optics switch using Mat lab simulation program (10). this technique includes design a mathematical model for analysis the effect of tensile $\operatorname{stress}\left(\tau_{\mathrm{y}}\right)$, axial $\left(\tau_{\mathrm{z}}\right)$ and radial $\left(\tau_{\mathrm{x}}\right)$ strain on the performance evaluation of an electro optic switch also, it analysis an effect the change of length $\Delta \mathrm{L}$ and width $\Delta \mathrm{w}$ of arm of switch. Finally, an active switch optimizes, using the analytical model and considers important device in the modern optical communication system.
\end{abstract}

Keywords: Laser source, Electro optic switch, tensile stress sensor, axial and radial strain sensor.

Paper History: Received: (28/9/2016), Accepted: (28/11/2016)

\section{Introduction}

With the ever quick improvement of optical fiber systems in nearby and trunk systems, there is a willing interest on cutting edge optical segments to completely use the colossal channel limit of optical fiber [1]. Thus, it is an inescapable pattern to create coordinated optoelectronic gadgets to build the data transmission, speed the reaction, and minimize the structure. Electro-optic switches have gotten to be vital parts of optical data change in optical communication systems because of their rapid and substantial limit [1-6]. In the course of recent years, it has turned into an earnest issue to outline and create optical switches with accurate components, for example, low switching voltage, short switching time, wide wavelength range, high extinction ratio, low polarization reliance, low insertion loss and cross talk, and high security. Likewise, awesome advancement has been made in the structure as well as in the materials. ${ }^{[7-10]}$ The sensible choice of electrooptic material is one of the powerful approaches to enhance the execution of electro-optic switches [1]. Contrasted and inorganic electro optic materials, polymer electro-optic materials have some phenomenal components, including high electro-optic coefficients, simple control of refractive index and basic innovation processing [11-14]. Electro-optic material like lithium niobate has refractive indices that can be changed by a connected electric field as appeared in the Figure 1. Numerous waveguide modulators or switches utilize metallic cathodes saved on top of optical waveguide to effectively apply the electric field [15]. A moderate buffer layer with low dielectric steady frequently saved between the anodes and the substrate to decrease the losses that are because of the metallic coating of the waveguide [1]. The proficiency of the gadget relies on upon cover between the electric field and the optical field [16].

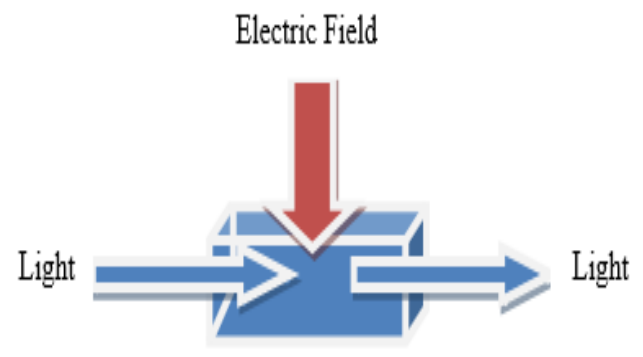

Figure 1: An electric field applied to an electro optic material altered its refractive indices

\section{Electro Optic Effect}

Mach Zehnder Interferometer (MZI) can be utilized as active optical switch if a voltage is connected as appeared in Figure 2 [16]. The constraint forced by direct current modulation of semiconductor injection lasers at present limits the most extreme achievable modulation frequencies to few giga hertzes [17]. Moreover, with most injection lasers fast current modulation additionally makes undesirable wavelength modulation which forces issues for systems utilizing wavelength division multiplexing. Along these lines to increase the data transfer capacity ability of single mode fiber systems there is a demand for high speed modulation which can be given by integrated optical waveguide intensity modulation. Basic on/off modulation might be construct be situated in light of the systems used for the dynamic bar splitters and switches. 
What's more a huge assortment of prevalently electro-optical modulation have been accounted for which show great qualities [16]. For instance, an essential waveguide modulator is based upon a Y-branch interferometer which utilizes optical phases moving created by the electro-optic impact [16]. The change in refractive index displayed by an electro-optic material with the use of an electric field given by $\delta_{\mathrm{n}}= \pm 5.0 \mathrm{n}^{3}{ }_{1} \mathrm{rE}$ likewise gives a phase change to light transmission in the material. This phase change $\delta \phi$ is collective over a separation L inside the material [16].

$$
\delta \phi=K \delta_{n} L
$$

At the point when the electric field is connected transversely to the course of optical spread we may substitute for $\delta_{\mathrm{n}}$ from $\delta_{\mathrm{n}}= \pm 5.0$ $\mathrm{n}^{3}{ }_{1} \mathrm{rE}$ giving ${ }^{[16]}$

$$
\delta \phi=\frac{\pi n_{1}^{3} r E L}{\lambda}
$$

Besides taking $\mathrm{E}$ equivalent to $\mathrm{V} / \mathrm{d}$, where $\mathrm{V}$ is the connected voltage and $\mathrm{d}$ is the separation between cathodes gives [16]

$$
\delta \phi=\frac{\pi n_{1}^{3} r V L}{\lambda d}
$$

It might be noted from $\delta \phi=\frac{\pi n_{1}^{3} r V L}{\lambda d}$ that so as to decrease the connected voltage $\mathrm{V}$ required to give a specific phases change, the proportion L/d must be made as expanded as could be expected under the condition [16]. A basic phase modulator may in this manner be acknowledged on a strip waveguide in which the proportion $\mathrm{L} / \mathrm{d}$ is expansive as appeared in Figure 3. These gadgets when, for instance, manufactured by spread of $\mathrm{Nb}$ into $\mathrm{LiNbO} 3$, give a change of $\pi$ radians with a connected voltage in the extent 5 to $10 \mathrm{~V}$, consequently gives optical switching [16]. The result of these no uniform fields can be combine into a cover fundamental a, having a worth somewhere around 0 and 1 which gives a measure of cover between the electrical and optical and optical fields. The electro-optic refractive index change of $\delta_{\mathrm{n}}= \pm 5.0 \mathrm{n}^{3}{ }_{1} \mathrm{rE}$ in this way gets to be [16].

$$
\delta \phi=\frac{a n_{1}^{3} r V}{2 d}
$$

Where the element a perform to the effectiveness of the electro-optical communication in respect to a romanticized parallel plate capacitor with the same separation between the cathodes. MachZehnder interferometer comprises of a couple of waveguides which are parallel of each other and isolated by a partition separation [16]. When electric field is applying, the refractive index of the electro-optic material like lithium niobate can be changed. Optical switches or modulators use cathode plate which situated on the coupling locale to couple the information optical signal. A cushion layer with low dielectric consistent is included between the anode and the substrate to lessen losses because of the cathode plate [19]. Coupling effectiveness of the optical switch outlined depends to a great extent on the covering of the optical field and electric field. With changing the anode parameters, the coupling effectiveness of the optical switch composed can be improved [16].

The property of electro-optic can be utilized in an interferometer intensity modulator. Such a Mach Zehnder interferometer is appeared in Figure 4 [16]. The gadget includes two Yintersections which give an equivalent division of the info optical power. With no potential connected to terminals, the info optical power is part into the two arms at the principal $\mathrm{Y}$ intersection and touches base at the second Yintersection in phase giving a power most extreme at the waveguide yield [20]. This condition compares to the "no" state. Then again when a potential is connected to the cathodes, which work in a push-pull mode on the two arms of the interferometer, a differential phase change is made between the signals in the arms [16]. The consequent recombination of the signals offers ascend to constructive or destructive interference in the yield waveguide. Thus the procedure has the impact of changing over the phase modulation into intensity modulation. A phase shift of a between the two arms gives the "off" state for the gadget. Fast interferometer modulators have been shown with titanium doped lithium niobate waveguide. A $1.1 \mathrm{GHz}$ modulation data transfer capacity has been accounted for a $6 \mathrm{~mm}$ interferometer utilizing a $3.8 \mathrm{~V}$ on/off voltage over a $0.9 \mu \mathrm{m}$ cavity. Comparable gadgets joining cathodes on one arm just might be used as switching and are by and large touched on to as adjusted bridge interferometric switches [16].

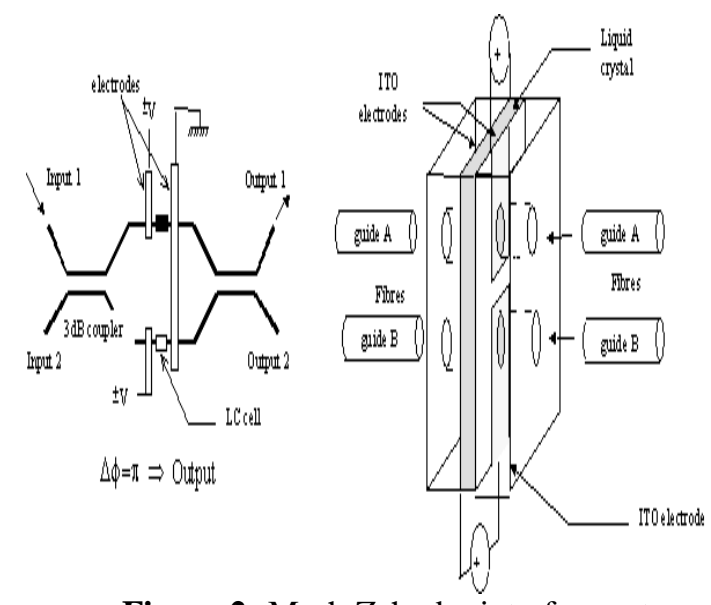

Figure 2: Mach Zehnder interferometer switch 


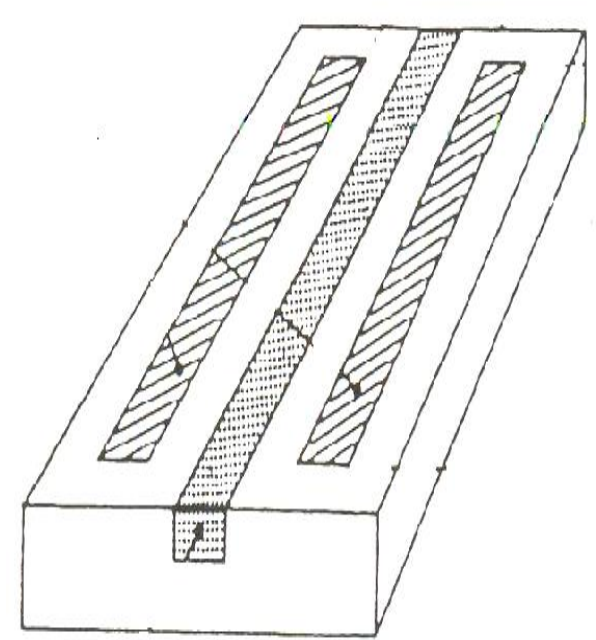

Figure 3: A simple strip waveguide phase modulator

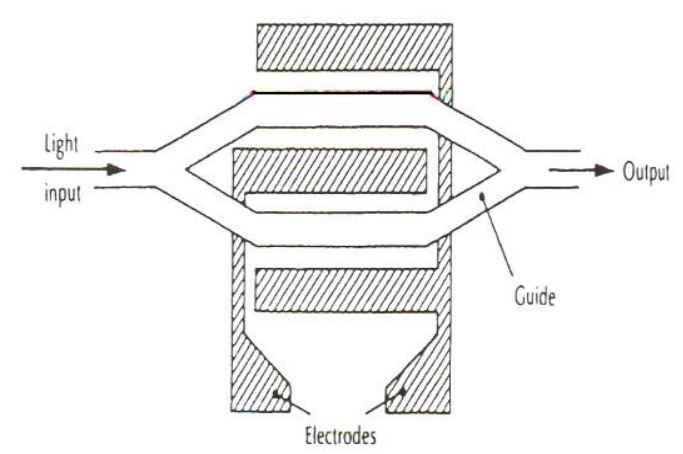

Figure 4: A Y-junction interferometric modulator based on the Mach Zehnder interferometer

\section{Mathmatical Model}

The equation (5), shows generally the equation of an optical interferometer switch when the incident light is split in two parts $\left(\mathrm{I}_{1}\right.$ and $\mathrm{I}_{2}$ ), which follow independent optical path and are merged again in a combiner where they interfere with each other So, when the laser diode is used then [21]:

$$
\begin{aligned}
\frac{2 \mathrm{I}_{1}-\mathrm{I}_{2}}{\mathrm{I}_{2}}=2 \mathrm{E}_{1} & {\left[\left(\mathrm{E}_{1}-1\right)\right.} \\
& +(1 \\
& \left.\left.-\mathrm{E}_{1}\right) \cos \Delta \emptyset\right]
\end{aligned}
$$

Where $I_{1}$ and $I_{2}$ are intensity of light for the arm one and arm two respectively and $\left(E_{1}\right)$, is electric field of the waveguide. So, if the voltage is applying on the electrodes as shown in Figures 5, a phase difference $\Delta \phi$ between the light propagation through the two waveguides will be introduced. and which presents an electro-optic active switch as shown in equation (2), then [16].

$$
E=\frac{2 \Delta_{n} V_{\pi} L}{\lambda d}
$$

Where $\left(\mathrm{V}_{\pi}\right)$, is switching voltage, $(\mathrm{L})$, is a length of the electrodes and (d), is a distance of the separation between the electrodes and $(\lambda)$, is an input wavelength.

So, Substituting Eq.(6), in to Eq. (5), yields:

$$
\begin{aligned}
\frac{2 \mathrm{I}_{1}-\mathrm{I}_{2}}{\mathrm{I}_{2}}=2\left(\frac{2 \Delta_{\mathrm{n}} \mathrm{V}_{\pi} \mathrm{L}}{\lambda \mathrm{d}}\right)\left[\left(\frac{2 \Delta_{\mathrm{n}} \mathrm{V}_{\pi} \mathrm{L}}{\lambda \mathrm{d}}-1\right)\right. \\
\left.+\left(1-\frac{2 \Delta_{\mathrm{n}} \mathrm{V}_{\pi} \mathrm{L}}{\lambda \mathrm{d}}\right) \cos \Delta \emptyset\right]
\end{aligned}
$$

$\frac{2 \mathrm{I}_{1}-\mathrm{I}_{2}}{\mathrm{I}_{2}}$

$=\left(\frac{4 \Delta_{\mathrm{n}} \mathrm{V}_{\pi} \mathrm{L}}{\lambda \mathrm{d}}\right)\left[\frac{2 \Delta_{\mathrm{n}} \mathrm{V}_{\pi} \mathrm{L}-\lambda \mathrm{d}+\left(\lambda \mathrm{d}-2 \Delta_{\mathrm{n}} \mathrm{V}_{\pi} \mathrm{L}\right) \cos \Delta \emptyset}{\lambda \mathrm{d}}\right]$

$$
\begin{aligned}
& \frac{2 \mathrm{I}_{1}-\mathrm{I}_{2}}{\mathrm{I}_{2}} \\
& =\left[\frac{8 \Delta_{n}^{2} V_{\pi}^{2} L^{2}-4 \Delta_{n} V_{\pi} L \lambda d+\left(4 \Delta_{n} V_{\pi} L \lambda d-8 \Delta_{n}^{2} V_{\pi}^{2} L^{2}\right) \cos \Delta \emptyset}{\lambda^{2} d^{2}}\right] \\
& \frac{\left(2 \mathrm{I}_{1}-\mathrm{I}_{2}\right)\left(\lambda^{2} \mathrm{~d}^{2}\right)}{\mathrm{I}_{2}}=8 \Delta_{\mathrm{n}}^{2} \mathrm{~V}_{\pi}^{2} \mathrm{~L}^{2}-4 \Delta_{\mathrm{n}} \mathrm{V}_{\pi} \mathrm{L} \lambda \mathrm{d} \\
& +\left(4 \Delta_{\mathrm{n}} \mathrm{V}_{\pi} \mathrm{L} \lambda \mathrm{d}\right. \\
& I_{2} \\
& \left.-8 \Delta_{\mathrm{n}}^{2} \mathrm{~V}_{\pi}^{2} \mathrm{~L}^{2}\right) \cos \Delta \emptyset \\
& =\frac{2 I_{1} \lambda^{2} d^{2}}{\lambda^{2} d^{2}+8 \Delta_{\mathrm{n}}^{2} V_{\mathrm{T}} L^{2}-4 \Delta_{\mathrm{n}} V_{\mathrm{d}} L \lambda d+\left(4 \Delta_{\mathrm{n}} V_{\mathrm{d}}\right.}
\end{aligned}
$$

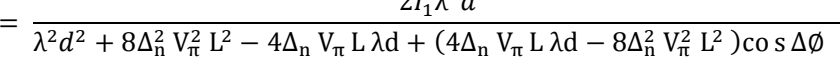

Equation (7) explains the relationship between the intensity of light of electro optic switch and the phase difference $\Delta \emptyset$ where the phase difference explain the parameter effects such as the force of stress and the tensile stress on the path of intensity of light for an electro optic switch .The phase difference $\Delta \emptyset$ is related by equation (8), [22].

$$
\begin{aligned}
& \Delta \emptyset=\frac{2 \pi n L}{\lambda}\left[\left(\frac{1}{n}\right)\left(\frac{\partial n}{\partial T}\right) \Delta T+\tau_{Z}[1\right. \\
& -\left(\frac{n^{2}}{2}\right)\left[\rho_{x y}+\rho_{y y}-v\right. \\
& \left.\left.+\rho_{y y}\right]\right] \\
& \tau_{z}=-\frac{\tau_{X}}{v} \\
& \tau_{z}=\frac{\tau_{y}}{G} \\
& A_{O}=\left(\frac{D}{2}\right)^{2} * \pi \\
& v=-\frac{\Delta w / w}{\Delta L / L} \\
& \Delta \emptyset=\frac{2 \pi n L}{\lambda}\left[\left(\frac{1}{n}\right)\left(\frac{\partial n}{\partial T}\right) \Delta T+\frac{\tau_{y}}{G}[1-\right. \\
& \left.\left(\frac{n^{2}}{2}\right)\left[\left(\rho_{x y}+\rho_{y y}\right)-\frac{\Delta w / w}{\Delta L / L}+\frac{n^{2}}{2} \rho_{y y}\right]\right]
\end{aligned}
$$

Equation (9) explains the difference of phase $(\Delta \phi)$. Where the stress, strain, the change of length $(\Delta \mathrm{L})$ and of width $(\Delta \mathrm{w})$ of an arm of electro optic switch considered in eq.(9). Where: $K=\frac{2 \pi}{\lambda}, \mathrm{K}=$ wave number, $\mathrm{L}=$ The length of the crystal arm in the electro optic $\operatorname{switch}(\mu \mathrm{m}),(\mathrm{d})$ is a distance of the separation 
between the electrodes, $\lambda=$ Free space wavelength of the laser in $(\mathrm{nm}), \mathrm{n}=$ Refractive index (unit less), $\Delta \mathrm{T}=$ Temperature difference in $(\mathrm{k}), \frac{\partial n}{\partial T}=$ thermo optic coefficient in $\mathrm{K}^{-1}, \tau_{\mathrm{x}}=$ radial strain in ( $\mu$-epsilon),$\tau_{\mathrm{z}}=$ axial strain in( $\mu$-epsilon $), \tau_{y}=$ Tensile stress in $\left(\mathrm{N} / \mathrm{mm}^{2}\right)$ and $\rho_{\mathrm{xy}}$ and $\rho_{\mathrm{yy}}=$ pocket coefficient , $\mathrm{G}=$ Young's modules in Gpa , $\boldsymbol{v}=$ Poisson's Ratio, $\Delta \mathrm{w}=$ the change in the width of crystal arm in $\mu \mathrm{m}, \mathrm{w}=$ the diameter of the crystal arm in $\mu \mathrm{m}$ and $\Delta \mathrm{L}=$ the change in the length of crystal arm in the $\mu \mathrm{m}$.

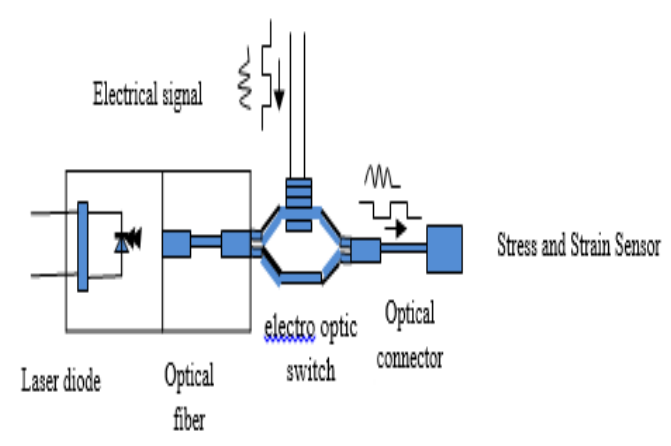

Figure 5: Illustration electro optic switch with tensile stress, axial and radial strain sensor

\section{Simulation and Results}

If a force in the form tensile stress is applied on an arm of an electro optic switch, the axial and radial strain will be produce and this result to change the length $\Delta \mathrm{L}$ and width $\Delta \mathrm{w}$ of an arm of switch as appeared in Figure 6, Table 1 and Table 2.

From Table 1, the force is applies from 50 into $550 \mathrm{MN}$ on the arm of optical switch where the length of an arm $\mathrm{L}$ is $10 \mathrm{~mm}$ and the tensile stress, axial strain and change of the length of an arm $\Delta \mathrm{L}$ increased gradually from 9.94718*18 $\mathrm{Mpa}, 0.09947$ ( $\mu$ epsilon) and $0.994 \mathrm{~mm}$ until its reach extreme value $1.09419 * 10^{5}$ Mpa,1.0941( $\mu$ epsilon) and (10.941) respectively.

Also, from the Table 2 when it applies the force from a value 50 into $2600 \mathrm{MN}$ on an arm's switch which the width of an arm (w) is $80 \mu \mathrm{m}$ so, the tensile stress, axial strain, radial strain i.e., (width strain), and the change of width of an arm $(\Delta w)$ are increased progressively from $9.94718 * 18^{3} \mathrm{Mpa}$, 1.0941( $\mu$ epsilon), $-1.989 * 10^{-2}$, and 1.591 into its extent extreme value $5.1725 * 10^{5} \mathrm{Mpa}$, 5.1725( $\mu$ epsilon), -1.0345 , and $82.76 \mu \mathrm{m}$ respectively. So, when $\Delta \mathrm{L}, \Delta \mathrm{w}$ extent to extreme value $10.941 \mathrm{~mm}$, and $82.76 \mu \mathrm{m}$ respectively, these leads to the arm of an electro optic switch will be an inactive and this due to, that an interference signal of an electro optic switch is removed because of the damaged of an electro optic switch. From all of this, it can be suggested a mathematical model where an equation (7), as shown in a previous section, to analysis these results and a damaged can be remove by using this technique.

From these Figures 7, 8 and 9, it can be noticed the waveform whose amplitude goes on decreasing gradually with time are called damping wave. So, when it applies the force the normalize intensity decreasing in the form damping wave and the power losses continuously with increasing the tensile stress, axial strain and radial strain as shown in Figures 7, 8 and in Figure 9, it may be noted that relation shows inversely because of the normalize intensity decreasing with increasing radial strain from 0 into -1.4. So, the performance of the electro optic switch affects with decreasing normalize intensity on the other hand increasing stress and strain. Otherwise, the efficiency of an electro optic switch is better. Also, from Fig.(10), when a force is increasing gradually the normalize intensity is decreasing into -20 and it results to decay of interference signal, addition to, from Figs. (11 and 12) when the $\Delta \mathrm{L}$ and $\Delta \mathrm{w}$ value is larger than $9 \mathrm{~mm}$ and $80 \mu \mathrm{m}$ respectively the normalize intensity is decreasing and this leads to weaken the interference signal otherwise if the value of $\Delta \mathrm{L}$ and $\Delta \mathrm{w}$ are less than $1 \mathrm{~mm}$ and $1 \mu \mathrm{m}$ equal to 0 respectively i.e. $(\Delta \mathrm{L}<1 \mathrm{~mm}$ and $\Delta \mathrm{w}<1 \mu \mathrm{m}$ or equal to 0 ), the normalize intensity is high and the performance of switch works with higher efficiency.

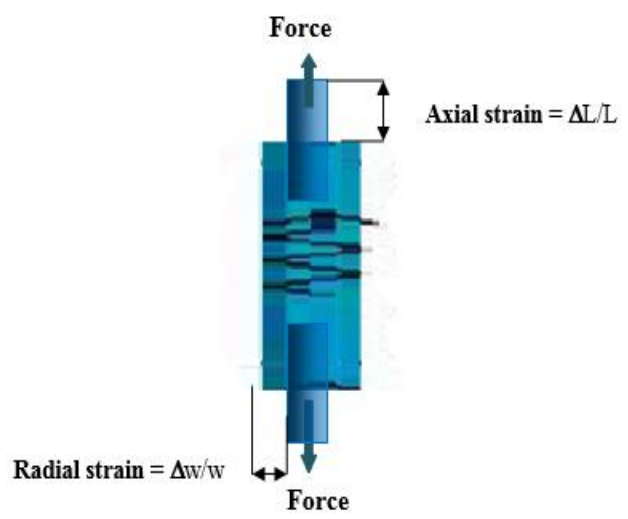

Figure 6: Illustration applying force on an arm of the electro optic switch 
Table 1

\begin{tabular}{|c|c|c|c|c|}
\hline Force $(\mathrm{M})$ & $\begin{array}{c}\text { tensile stress } \\
\left(\tau_{\mathrm{r}}=\mathrm{F} / \mathrm{Ao}\right)(\mathrm{Mpa})\end{array}$ & $\begin{array}{l}\text { axial strain } \\
\left(\mathrm{r}=\mathrm{r}_{\mathrm{y}} / \mathrm{G}\right)\end{array}$ & $\mathrm{L}(\mathrm{mm})$ & $\mathbb{\Delta L}(\mathrm{mm})$ \\
\hline 50 & $9.94718^{*} 10^{3}$ & 0.09947 & \multirow{6}{*}{$\frac{1}{10 \mathrm{~mm}}$} & 0.994 \\
\hline 150 & $2.98411^{*} \times 0^{4}$ & 0.2984 & & 2.984 \\
\hline 250 & $4.97359 * 10^{4}$ & 0.4973 & & 4.973 \\
\hline 350 & $6.96302 * 10^{4}$ & 0.6963 & & 6.963 \\
\hline 450 & $8.95240^{*} 10^{4}$ & 0.8952 & & 8,952 \\
\hline 550 & $1,09411^{*} 10^{2}$ & 1.0941 & & 10.941 \\
\hline
\end{tabular}

Table 2

\begin{tabular}{|c|c|c|c|c|c|}
\hline Force $(\mathrm{N})$ & $\begin{array}{l}\text { tensile stress } \\
\left(\tau_{\mathrm{\tau}}=\mathrm{F} / \mathrm{A}_{0}\right) \\
(\mathrm{M} \mathrm{pa})\end{array}$ & $\begin{array}{l}\text { axial strain } \\
\left(\tau_{z}=\tau_{\digamma} / G\right)\end{array}$ & $\begin{array}{c}\text { radial strain } \\
\left(\tau_{x}=-v \tau_{z}\right)\end{array}$ & $w(\mu \mathrm{m})$ & $\Delta w(\mu \mathrm{m})$ \\
\hline 50 & $9.94718^{8} 10^{3}$ & 0.09947 & $-1.989 * 10^{-2}$ & \multirow{23}{*}{${ }^{80 \mu \mathrm{m}}$} & 1.591 \\
\hline 150 & $2.98415 * 10^{4}$ & 0.2984 & $-5.968 \times 10^{-2}$ & & 4.774 \\
\hline 250 & $4.97359 \times 10^{4}$ & 0.4973 & $-9.946 * 10^{-2}$ & & 7.956 \\
\hline 350 & $6.96302 \times 10^{4}$ & 0.6963 & $-1.392 \times 10^{-1}$ & & 11.136 \\
\hline 450 & $8.95246^{*} 10^{4}$ & 0.8952 & $-1.790 * 10^{-1}$ & & 14.32 \\
\hline 550 & $1.09419 \times 10^{2}$ & 1.0941 & $-2.188 \div 10^{-1}$ & & 17.504 \\
\hline 650 & $1.29313 * 10^{2}$ & 1.29313 & $-2.586 * 10^{-1}$ & & 20.688 \\
\hline 750 & $1.49207 \times 10^{2}$ & 1.49207 & $-2.984 \times 10^{-1}$ & & 23.87 \\
\hline 850 & $1.6910_{2}^{2} \times 10^{2}$ & 1.69102 & $-3,382 * 10^{-1}$ & & 27.056 \\
\hline 950 & $1.88996 * 10^{2}$ & 1.88996 & $-3.779 \times 10^{-1}$ & & 30.232 \\
\hline 1050 & $2.08890^{\circ} \times 10^{2}$ & 2.08890 & $-4.1778 \div 10^{-1}$ & & 33.422 \\
\hline 1150 & $2.28785^{*} \times 10^{2}$ & 2.28785 & $-4.575^{*} 10^{-1}$ & & 36.6 \\
\hline 1250 & $2.48679 \times 10^{2}$ & 2.48679 & $-4.973^{*} \times 10^{-1}$ & & 39.784 \\
\hline 1350 & $2.68573 \times 10^{2}$ & 2.68573 & $-5.371 \times 10^{-1}$ & & 42.968 \\
\hline 1450 & $2.88468 * 10^{2}$ & 2.88468 & $-5.769 * 10^{-1}$ & & 46.152 \\
\hline 1550 & $3.08362 \times 10^{2}$ & 3.08362 & $-6.1724 \times 10^{-1}$ & & 49.37 \\
\hline 1650 & $3.2857^{7} 10^{2}$ & 3.28257 & $-6.565 \mathrm{I}^{1} 10^{-1}$ & & 52.52 \\
\hline 1750 & $3.48151 \times 10^{2}$ & 3.48151 & $-6.963 * 10^{-1}$ & & 55.704 \\
\hline 1850 & $3.68045^{*} \times 10^{2}$ & 3.68045 & $-7.3600 \times 10^{-1}$ & & 58.8 \\
\hline 1950 & $3.87940 \times 10^{2}$ & 3.87940 & $-7.758 \times 10^{-1}$ & & 62.064 \\
\hline 2200 & $4.3767 \times 10^{7}$ & 4.3767 & $-8.753 * 10^{-1}$ & & 70.024 \\
\hline 2400 & $4.474^{* 10^{2}}$ & 4.474 & -0.89 & & 78.4 \\
\hline 2600 & $5.1725^{*} 10^{2}$ & 5.1725 & -1.0345 & & 82.76 \\
\hline
\end{tabular}

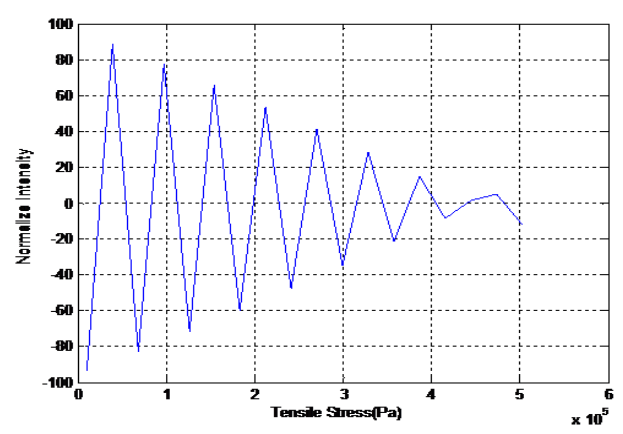

Figure 7: The tensile stress verses of normalize intensity

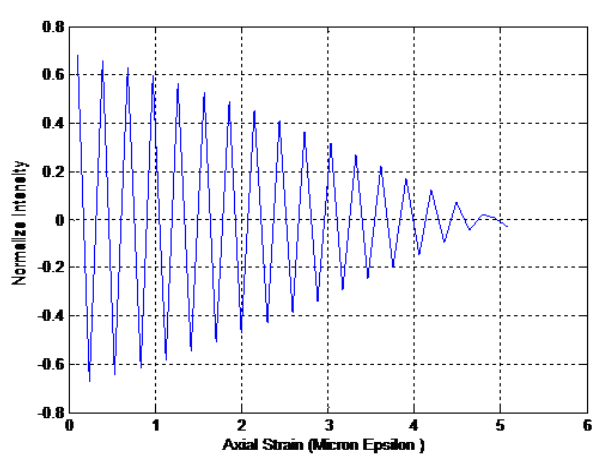

Figure 8: The axial strain verses of normalize intensity

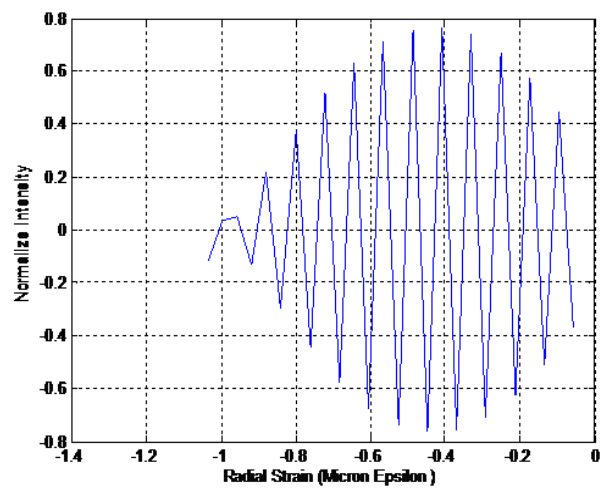

Figure 9: The radial strain verses of normalize intensity

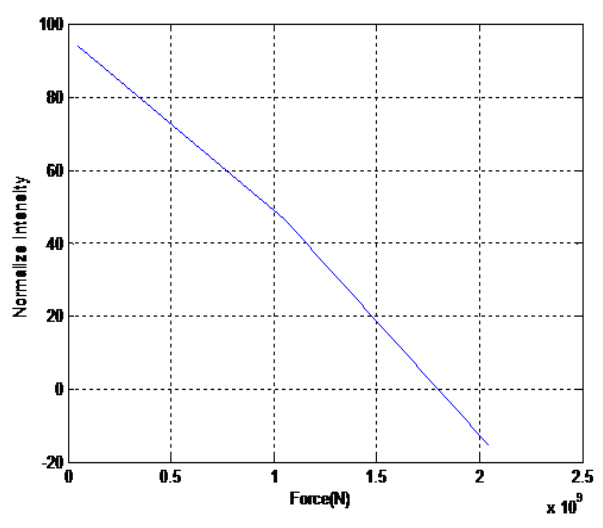

Figure 10: The Force verses of normalize intensity 


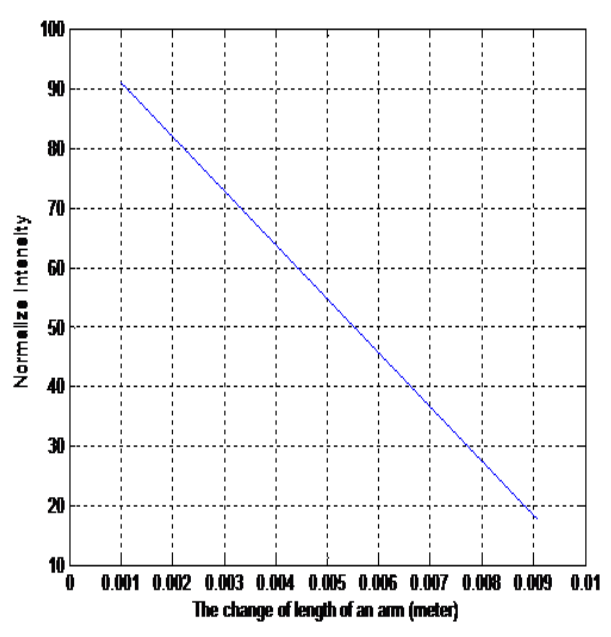

Figure 11: The change of the length $(\Delta \mathrm{L})$ of an arm verses of normalize intensity

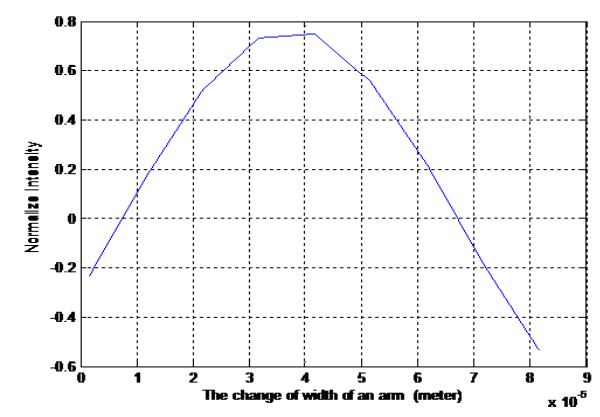

Figure 12: The change of the width $(\Delta \mathrm{w})$ of an arm verses of normalize intensity

\section{Conclusions}

In this research, we introduced a mathematical model is an effective way to improve the electro-optic switch sensitivity when the normalize intensity is relatively high and it can improve electro optic switch performance by reducing an effect of the tensile stress, axial and radial strain. We discussed the impact of increasing stress and strain effect on the performance of electro optic switch therefore it can be concluded when the stress and strain increase the normalize intensity decreases in the form damping wave and this leads to losses of power therefore this leads to decreasing a performance of electro optic switch thus it results a damage the arm of electro optic switch. We concluded the performance of electro optic switch can be improved by decreasing the change of the length $\Delta \mathrm{L}$ and width of an arm $\Delta \mathrm{w}$ i.e. . ( $\Delta \mathrm{L}<1 \mathrm{~mm}$ and $\Delta \mathrm{w}<1 \mu \mathrm{m}$ or equal to 0 ), which result from increasing the axial and radial strain therefore they limited by controlling on the axial and radial strain. Finally, we presented a mathematical model it has ability to suppress the effect of stress and strain by evaluating its effects (i.e. $\mathrm{F}<50 \mathrm{MN}$ ).

\section{References}

[[1]. Chuan-Tao Zheng, Chun-Sheng Ma, Xin Yan, Xian-Yin Wang, Da-Ming Zhang, Design and analysis of a polymer Mach- Zehnder interferometer electro-optic switch over a wide spectrum of $110 \mathrm{~nm}$, Optical Engineering 48(5), 054601, May (2009).

[[2]. Q. Wang and J. P. Yao, "A high speed 2x2 electrooptic switch using a polarization modulator," Opt. Express 15(25), 16500-16505 (2007).

[[3]. H. Muller, S. W. Chiow, S. Herrmann, and S. Chu, Nanosecond electro-optical switching with a repetition rate above $20 \mathrm{MHz}$, Rev. Sci. Instrum. 78(12), 124702 (2007).

[[4]. E. Sarailou, A. Gharavi, S. Javadpour, and V. Shkunov, Grating based electro-optic switch with azo nonlinear optical polymers, Appl. Phys. Lett. 89(17), 171114 (2006).

[[5]. Y. Tanushi and S. Yokoyama, Design and simulation of ring resonator optical switches using electro-optic materials, Jpn. J. Appl. Phys., Part 1 45(4B), 3493-3497 (2006).

[[6]. Matthew Wade Puckett, Generating, Enhancing, and Leveraging Nonlinear and Electro-Optic Effects in Silicon- Based Waveguides, Doctor of Philosophy in Electrical Engineering (Photonics), university of California, san diego, 2016.

[[7]. H. Jung, 2x2, 1x4 Ti:LiNbO3 digital optical switches, Opt. Eng. 6(3)

[[8]. K. Suzuki, T. Yamada, M. Ishii, T. Shibata, and S. Mino, High-speed optical switch based on generalized Mach-Zehnder interferometer with hybrid configuration of silica-based PLC and lithium niobate phase-shifter array," IEEE Photonics Technol. Lett. 19(9-12), 674-676 (2007).

[[9]. Y. Tian, X. S. Xiao, S. M. Gao, and C. X. Yang, All-optical switch based on two-pump four-wave mixing in fibers without a frequency shift, Appl. Opt. 46(23), 5588-5592 (2007).

[[10]. Y. Y. Lin, S. T. Lin, G. W. Chang, A. C. Chiang, Y. C. Huang, and Y. H. Chen, Electro-optic periodically poled lithium niobate Bragg modulator as a laser Q- switch, Opt. Lett. 32(5), 545-547 (2007).

[[11]. A. Gardelein, T. S. Le, E. Tanguy, N. Breuil,, and T. Razban- Haghighi, Passive electro-optic antenna using polymer material, Electron. Lett. 43(9), 489491 (2007).

[12] R. Swamy, H. Rajagopalan, P. Vippa, M. Thakur, and A. Sen, Quadratic electro-optic effect in a nano-optical material based on the nonconjugated conductive polymer, poly_ethylenepyrrolediyl_ derivative, Solid State Commun. 143(11)12, (519)521 (2007).

[13]. H. Rajagopalan, P. Vippa, and M. Thakur, Quadratic electro-optic effect in a nano-optical 
material based on the nonconjugated conductive polymer, poly_beta-pinene, Appl. Phys. Lett. 88(3), 033109 (2006).

[14]. X. X. Deng, P. P. Xiao, X. Zheng, Z. Q. Cao, Q. S. Shen, K. Zhu, H. G. Li, W. Wei, S. X. Xie, and Z. J. Zhang, An electro-optic polymer modulator based on the free-space coupling technique, J. Opt. A, Pure Appl. Opt. 10(1), 015305 (2008).

[15]. Alexandar Davis Dupuy, Processing and Properties of High Performance Lead Free Electro-Optic Ceramics, Doctor of Philosophy in Mechanical Engineering, university of California riverside, August, 2016.

[16]. ESMAEL .H .M YAHYA MACH-ZEHNDER INTERFEROMETER, MACH- ZEHNDER INTERFOMETER SWITCH, Master of Engineering(ElectricalElectronic\&Telecommunicat ion)Faculty of Electrical Engineering University Technology Malaysia, APRIL, 2007.

[17]. Deepak Venkatesh Simili, NANOPHOTONIC SILICON ELECTRO-OPTIC SWITCH, the degree of Master of Applied Science at Dalhousie University Halifax, Nova Scotia, August (2012).

[18]. Rajdeep Gautam, Silicon Microring Resonator Loaded Mach- Zehnder Interferometer for Low Power Optical Modulation, Switching and BioSensing" Department of Physics, Electrical \& Computer Engineering, Doctoral Thesis at Yokohama National University, March, 2014.

[19]. Mark R. Woolston, FAST ELECTRONIC DRIVER FOR OPTICAL SWITCHES Department of Electrical and Computer Engineering,The Degree of Master of Science, Colorado State University, Spring, 2012.

[20]. Yufei Xing, Low power optical switching based on slot waveguide infiltrated with liquid crystal European Master of Science in Photonics, Ghent University, (2013).

[21]. Wei-Chih Wang, Phase modulation" Southern Taiwan University of Technology.

[22]. D.D. Johnson MatSE 280: Introduction to Engineering Materials, (2010) . 